\title{
Quantum Computing of Poincaré Recurrences and Periodic Orbits
}

\author{
B. Georgeot \\ Laboratoire de Physique Théorique, UMR 5152 du CNRS, IRSAMC, Université Paul Sabatier, 31062 Toulouse Cedex 4, \\ France
}

(July 30, 2003)

\begin{abstract}
Quantum algorithms are built enabling to find Poincaré recurrence times and periodic orbits of classical dynamical systems. It is shown that exponential gain compared to classical algorithms can be reached for a restricted class of systems. Quadratic gain can be achieved for a larger set of dynamical systems. The simplest cases can be implemented with small number of qubits.
\end{abstract}

PACS numbers: 03.67.Lx, 05.45.Ac, 05.45.Tp

It has been suggested since Feynman [1] that the superposition principle of quantum mechanics enables to perform exponentially many computations in parallel (see reviews in $[2-4]$ ). Thus in principle quantum processors using the full power of quantum mechanics may be enormously faster than classical nowadays computers. This possibility has motivated a great deal of attention in the scientific community, and many experimental proposals are explored to realize such a quantum computer. However, it has been surprisingly hard to spot specific problems where quantum algorithms may be faster than classical ones. One such example is the celebrated Shor's algorithm [5] which factors large numbers with exponential efficiency compare to any known classical algorithm. Another algorithm, due to Grover [6], speeds up the search in an unsorted database, although not exponentially.

In parallel, people have investigated the possibility of using a quantum device to simulate physical systems, a problem of much practical interest. In particular, several works have shown that quantum computers can speed up the simulation of quantum mechanical systems [7-9] and classical spin systems [10]. It was also suggested in [11] that a quantum computer may be efficient at simulating classical dynamical systems as well. In the present work, the problem of finding Poincaré recurrence times and periodic orbits of dynamical systems is studied. Such quantities give important information about the dynamics, and are standard tools for studying classical mechanics. Explicit algorithms will be presented showing that such quantities can be evaluated faster on a quantum computer than on a classical one. In some cases, exponential efficiency compared to classical algorithms can be reached. In other cases, quadratic gains can be realized.

For classical bounded conservative systems, Poincaré [12] showed that some points from an arbitrary small phase space domain will eventually come back to this domain. In general, actual values of the recurrence time for a given area of phase space, and statistics of these times, are not given by the theorem and require specific studies to be understood. This time can be extremely long, and is very hard to study numerically. Advances in the power of modern classical computers allow to get information about such quantities, but the problem is still under investigation nowadays [13]. In addition to their intrinsic interest, the return times give also insight on transport properties, diffusion coefficients and correlation functions of the system studied [13]. A related feature of dynamical systems is the presence of periodic orbits, which are trajectories coming back exactly to their initial point in phase space. They play a special role in understanding physical properties of these systems, and are present both in Hamiltonian and dissipative systems. For example, they enable to compute diffusion coefficients and properties of strange attractors [14], and enter the famous Gutzwiller trace formula [15] which is a general semiclassical quantization scheme valid for chaotic systems.

I will first show how such quantities can be found efficiently on a particular example, the Arnold cat map $[16,17]$, one of the most famous chaotic dynamical systems. It is an automorphism of the torus with equation:

$$
\bar{y}=y+x(\bmod 1), \bar{x}=y+2 x(\bmod 1),
$$

where bars denote the new values of the variables after one iteration. This map is area-preserving, and may be interpreted as describing the evolution in phase space, with $x$ representing position and $y$ momentum. It is an Anosov system, with homogeneous exponential divergence of trajectories and positive Kolmogorov-Sinai entropy $h \approx 0.96$. The map (1) can be equivalently written as action of the $2 \times 2$ matrix $L=\left(\begin{array}{ll}2 & 1 \\ 1 & 1\end{array}\right)$ on $\left(\begin{array}{l}x \\ y\end{array}\right)$. Other cat maps can be built by taking any $2 \times 2$ matrix $L$ with integer entries and determinant 1 .

It is known [18-20] that for the cat map (1) periodic orbits are in one-to-one correspondence with points with rational coordinates. Still, it is a computationally hard problem to find the period of a given point. An usual way for studying this problem is to consider the set of rational points sharing the same denominator $g$. These points form a $g \times g$ lattice in the phase space, which is invariant under the action of the map. The action of the map on such points can be written on the numerators only, namely: $\bar{y}=y+x(\bmod g), \bar{x}=y+2 x(\bmod g)$, 
or $\left(\begin{array}{c}\bar{x} \\ \bar{y}\end{array}\right)=L\left(\begin{array}{l}x \\ y\end{array}\right)(\bmod g)$, with $x, y, \bar{x}, \bar{y}$ integers.

All points in such a lattice are periodic, but not with the same period. A quantity similar to recurrence time is the lattice period function $\alpha(g)$. It is the smallest integer such that after $\alpha(g)$ iterations all points in the lattice have come back to the initial position, i.e. it is the recurrence time of the whole lattice. This quantity has been the subject of many studies [18-20], since it describes the whole set of periodic orbits of the system, and also gives insight on the quantization of the cat map $[18,20]$. In particular, the behavior of $\alpha(g)$ controls the ergodicity of the eigenfunctions of the quantum cat map for $\hbar=1 / g$ [21]. It turns out to be a very erratic function of $g$; some results on its statistical properties have been derived nonrigorously based on assumptions from probabilistic number theory [20]. Numerical checks for moderate values of $g$ show that the asymptotic régime is very slowly reached [20]. Thus for example computing $\alpha(g)$ in small windows around large values of $g$ will enable to check its asymptotic properties. This has interest both for number theory and study of dynamical systems.

A quantum computer of moderate size is able to compute the function $\alpha(g)$ for exponentially large values of $g$. One does not need to actually simulate the evolution of individual points in the lattice, since $\alpha(g)$ is the smallest integer $t$ such that $L^{t}=I(\bmod g)$, with $I$ the identity. Nine registers of $n_{q}$ qubits with $n_{q} \sim \log _{2} g$ are needed, one holding the values of time, the next four the matrix entries, and the last ones being workspace. The algorithm applies period-finding (which is the basis of Simon's [22] or Shor's [5] algorithms) to the four registers encoding the entries of the matrix. First one prepares the initial state, which is $N^{-1 / 2} \sum_{t=0}^{N-1}|t\rangle|1\rangle|0\rangle|0\rangle|1\rangle$ where $N=2^{n_{q}}$. It is easily built from the ground state of the system by applying $n_{q}$ Hadamard gates and 2 singlequbit flips. Then one constructs the time evolution to end up with the entries of the matrix $L^{t}$ modulo $g$ in the last four registers. To this aim, first the entries $\left(a_{i}, b_{i}, c_{i}, d_{i}\right)$ of each $L^{2^{i}}$ for $i=1, \ldots, n_{q}-1$ are precomputed classically. This can be done sequentially, first squaring $L$ to get $L^{2}$, then squaring $L^{2}$ and so on until $L^{2^{n_{q}-1}}$ is obtained. This requires of the order of $O\left(n_{q}^{3}\right)$ classical operations. For each $t$, the binary decomposition of $t$ is $t=\sum_{i=0}^{n_{q}-1} \alpha_{i} 2^{i}$ with $\alpha_{i}=0,1$, so that $L^{t}=\Pi_{i=0}^{n_{q}-1}\left(L^{2^{i}}\right)^{\alpha_{i}}$. So the exponentiation of $L$ can be done by $n_{q}$ multiplication by $L^{2^{i}}$ conditioned on the value of the qubit $i$. This matrix multiplication can be done by a sequence of number multiplication. For example, the sequence of transformations for the first two entries is: $N^{-1 / 2} \sum_{t=0}^{N-1}|t\rangle|a\rangle|b\rangle|c\rangle|d\rangle$

$\rightarrow N^{-1 / 2} \sum_{t=0}^{N-1}|t\rangle|a\rangle|b\rangle|c\rangle|d\rangle|a\rangle|b\rangle$ (copy)

$\rightarrow N^{-1 / 2} \sum_{t=0}^{N-1}|t\rangle\left|a a_{i}\right\rangle\left|b d_{i}\right\rangle|c\rangle|d\rangle\left|a b_{i}\right\rangle\left|b c_{i}\right\rangle$

$\rightarrow N^{-1 / 2} \sum_{t=0}^{N-1}|t\rangle\left|a a_{i}+b c_{i}\right\rangle\left|a b_{i}+b d_{i}\right\rangle|c\rangle|d\rangle\left|a b_{i}\right\rangle\left|b c_{i}\right\rangle$. This needs a controlled multiplier modulo $g$, which can be done following the procedure in [23] using two extra registers as workspace. To erase the unwanted registers one uses a standard trick: one builds the sequence of gates corresponding to $F:|u\rangle|v\rangle|0\rangle|0\rangle \rightarrow$ $|u\rangle|v\rangle\left|d_{i} b_{i} u-c_{i} b_{i} v\right\rangle\left|a_{i} c_{i} v-b_{i} c_{i} u\right\rangle$. Applying $F^{-1}$ to the state above sets the work registers to zero. One then does again the same operation for the next two entries of the matrix. This needs $O\left(n_{q}^{2}\right)$ operations, and should be done for all qubits $i$ in the first register to build $L^{t}$, so $O\left(n_{q}^{3}\right)$ gates are used in total. One ends up with the state $N^{-1 / 2} \sum_{t=0}^{N-1}|t\rangle\left|A_{t}\right\rangle\left|B_{t}\right\rangle\left|C_{t}\right\rangle\left|D_{t}\right\rangle$ where $\left(A_{t}, B_{t}, C_{t}, D_{t}\right)$ are entries of the matrix $L^{t}$ modulo $g$.

Then as in Shor's algorithm one performs a Quantum Fourier Transform (QFT) of the first register. This quantum analog of the classical Fourier transform needs $O\left(n_{q}^{2}\right)$ elementary gates to be performed. Since the last four registers describe a periodic function of $t$, peaks in the Fourier transform can be measured and yield the period $\alpha(g)$. Indeed, if $\alpha(g)$ divides $N$, then peaks are precisely at values of multiples of $N / \alpha(g)$, while otherwise broader peaks will appear but significant probability will be concentrated on the best rational approximants of multiples of $N / \alpha(g)$, and a continued fractions algorithm can yield the period in $O\left(n_{q}^{3}\right)$ classical operations [2-4].

It is worth trying to compare this algorithm to compute $\alpha(g)$ with others. Classically, computing all iterates $L^{k}$ up to the period is clearly exponential since $\alpha(g)$ is of the same order of magnitude as $g(\langle\alpha(g) / g\rangle \rightarrow 0$ slower than any inverse power of $g[20])$. Another method to compute $\alpha(g)$ [19] is based on the theory of ideals of quadratic fields. For a prime $p, \alpha(p)=\frac{p-(d / p)}{m}$, where () is the Kronecker symbol $(= \pm 1), d$ is the discriminant of the field containing the eigenvalues of $L$, and $m$ is an integer divisor of $p-(d / p)$ to be determined by trial and errors. For composite $g, \alpha(g)$ can be determined from the $\alpha(p), p$ being the prime factors of $g$. This method is also exponential classically, since it requires the factorization of $g$ in prime factors. On a quantum computer, one may try to use Shor's algorithm, first factoring $g$, then factoring $p-(d / p)$ for all prime factors of $g$ and trying all possible integer divisors. Most integers $g$ have $\sim \log \log g$ prime factors and for most $p$ there are $\sim(\log p)^{\log 2}$ divisors of $p-(d / p)[24]$. Thus this algorithm is also polynomial for most numbers. Still, it is known [24] that the number of divisors of an integer $n$ can be quite large, of the order of $2^{\log n / \log \log n}$ in worst cases, making this method not polynomial for some $g$. The algorithm here is simpler, giving $\alpha(g)$ in one run, and always polynomial.

Another possibility to explore periodic orbits and Poincaré recurrences which can be generalized to other systems beyond (1), is to start from an initial point and look for periodicities of its iterates. In this case, it is appropriate to use a discretized map. As discussed in [11], the best possible discretization uses symplectic maps [25]. These maps can be exactly iterated and are themselves 
Hamiltonian if the original system is. In dimension 2, they can be written as the action of a unitary operator $L$ on the $N^{2}$ points $\left(x_{i}, y_{j}\right)$ with $x_{i}=i / N, i=0, \ldots, N-1$ and $y_{j}=j / N, j=0, \ldots, N-1$, with $N=2^{n_{q}}$. For example, for the cat map, a point $\left(x_{i}, y_{j}\right)$ of the discretized phase space density is mapped to $\left(\bar{x}_{i}, \bar{y}_{j}\right)$ through the action of the $2 \times 2$ matrix $L$ defined above. The algorithm requires three registers, two of size $n_{q}$ specifying points in phase space and one of size $p$ holding the values of time, plus additional qubits as workspace, with usually $p \approx n_{q}$. One starts from the initial state $2^{-p / 2} \sum_{t=0}^{2^{p}-1}|t\rangle\left|x_{0}\right\rangle\left|y_{0}\right\rangle$, easily built from the action of $p$ Hadamard gates and $2 n_{q}$ single-qubit flips. Then one constructs a sequence of gates giving $2^{-p / 2} \sum_{t=0}^{2^{p}-1}|t\rangle\left|L^{t}\left(x_{0}\right)\right\rangle\left|L^{t}\left(y_{0}\right)\right\rangle$. Then a QFT of the first register will yield peaks, from which the period of the point can be found. If $L$ and each $L^{2^{k}}$ for $k=1, \ldots, p-1$ can be computed and implemented in $\left(O\left(B\left(n_{q}\right)\right)\right.$, where $B$ is a polynomial, this will be exponentially faster than classically. For the cat map, all can be done efficiently by a method similar to the one described above, first precomputing classically the $L^{2^{k}}$, then decomposing each $t$ in binary representation, and then using a controlled multiplier modulo $N$ to compute $\left(L^{t}\left(x_{0}\right), L^{t}\left(y_{0}\right)\right)$. Again, this uses $O\left(n_{q}^{3}\right)$ quantum gates.

Other maps can be explored in the same way, provided their classical evolution operator $L$ enables fast (polynomial) classical computation of $\left(L^{2^{k}}\right)$. In such cases, the algorithm above can reach exponential gain over the classical computation. If $L$ is efficiently implementable, but exponentially large iterates cannot be efficiently computed (see examples below) the algorithm above will not work. Nevertheless, in this case it is possible to obtain a gain for the computation of periodic orbits and recurrence properties of the whole system for a given time (as opposed to a chosen initial point). The basic idea is to compute sequentially the iterates of a distribution and then use Grover iterations to search for specific trajectories. The dynamical system is discretized through a symplectic map $L$, on a lattice of size $N \times N$ where $N=2^{n_{q}}$, and it is assumed that $L$ can be implemented on these $N^{2}$ points in $O\left(B\left(n_{q}\right)\right)$ operations where $B$ is a polynomial. Then a subdomain $A$ is selected. The simplest case is a square of size $P \times P$ with $P=2^{p}$ and $p<n_{q}$. The initial state is $\left|\psi_{0}\right\rangle=2^{-p} \sum_{i=0}^{2^{p}-1} \sum_{j=0}^{2^{p}-1}\left|x_{i}\right\rangle\left|y_{j}\right\rangle$. Then one realizes the transformation $\left|\psi_{0}\right\rangle \rightarrow 2^{-p} \sum_{i=0}^{2^{p}-1} \sum_{j=0}^{2^{p}-1}\left|L\left(x_{i}\right)\right\rangle\left|L\left(y_{j}\right)\right\rangle$ $\ldots \rightarrow 2^{-p} \sum_{i=0}^{2^{p}-1} \sum_{j=0}^{2^{p}-1}\left|L^{t}\left(x_{i}\right)\right\rangle\left|L^{t}\left(y_{j}\right)\right\rangle$. The value of the first $n_{q}-p$ qubits of each register is enough to know if the trajectory is back to the domain $A$ (since $A$ can be moved efficiently to a corner). So after $t$ iterations this value is checked for all trajectories and a $\sigma_{z}$ controlled by the value of these $2 n_{q}-2 p$ qubits gives a minus sign to the values of $\left|L^{t}\left(x_{i}\right)\right\rangle\left|L^{t}\left(y_{j}\right)\right\rangle$ which end a trajectory which returns to $A$. This can be done ef- ficiently using $O\left(\left(2 n_{q}-2 p\right)^{2}\right)$ elementary gates and no work qubit [4]. Then one inverts all the gates but the last operation, ending with $2^{-p} \sum_{i=0}^{2^{p}-1} \sum_{j=0}^{2^{p}-1} \epsilon\left|x_{i}\right\rangle\left|y_{j}\right\rangle$, where $\epsilon= \pm 1$ depending on the fact that the trajectory went back to $A$ after these $t$ iterations. This whole procedure is then used as an oracle for iterations of the Grover algorithm [6]. After $P / \sqrt{M}$ iterations, where $M$ is the number of solutions, the amplitude of the wavefunction is concentrated on initial points which return to $A$ after $t$ iterations, and a measure of the registers will give one of them. The whole algorithm necessitates $2 n_{q}$ qubits plus the workspaces. Only $O(t P / \sqrt{M})$ operations are needed to get the returns, as opposed to $O\left(t P^{2} / M\right)$ for the classical simulation (up to logarithmic factors). In $O(t P / \sqrt{M})$ one initial point of a trajectory which returns to $A$ is obtained. To get other trajectories which return, or for different return times, one needs to restart the algorithm. This process has obviously interest mostly for $t \ll P$. The number of returning trajectories $M$ can be evaluated through the quantum counting algorithm (phase estimation combined with the Grover iterations) in $O(t P)$ operations [26]. Since generally the number $M$ is unknown, one can estimate it by quantum counting prior to the search, but it is not necessary [26].

The algorithm can be modified to get a different result, which is the number and coordinates of the periodic orbits of the system. In this case one starts from all the $N \times N$ points of the lattice with $N=2^{n_{q}}$, with initial state $\left|\psi_{0}\right\rangle=$ $2^{-n_{q}} \sum_{i=0}^{2^{n} q}-1 \sum_{j=0}^{2^{n_{q}}-1}\left|x_{i}\right\rangle\left|y_{j}\right\rangle$. Then one performs: $\left|\psi_{0}\right\rangle \rightarrow 2^{-n_{q}} \sum_{i=0}^{2^{n_{q}}-1} \sum_{j=0}^{2^{n_{q}}-1}\left|x_{i}\right\rangle\left|y_{j}\right\rangle\left|L\left(x_{i}\right)\right\rangle\left|L\left(y_{j}\right)\right\rangle \quad \ldots \rightarrow$ $2^{-n_{q}} \sum_{i=0}^{2^{n} q}-1 \sum_{j=0}^{2^{n_{q}}-1}\left|x_{i}\right\rangle\left|y_{j}\right\rangle\left|L^{t}\left(x_{i}\right)\right\rangle\left|L^{t}\left(y_{j}\right)\right\rangle$. After $t$ iterations the value of the iterate is compared to the initial value (for example by adding the registers bitwise modulo 2 ), and a minus sign is given (by using a $\sigma_{z}$ controlled by the value of these qubits [4]) if it is the same. Then one invert all the gates but the last operation, ending with $2^{-n_{q}} \sum_{i=0}^{2^{n} q}-1 \sum_{j=0}^{2^{n} q}-1 \epsilon\left|x_{i}\right\rangle\left|y_{j}\right\rangle$, where $\epsilon= \pm 1$ depending on the fact that the trajectory is periodic of period $t$ or not. This whole procedure is then used as an oracle for iterations of the Grover algorithm [6] and all periodic orbits can be found by iterating the process. After $O(t N / \sqrt{M})$ operations, the amplitude of the wavefunction is concentrated on periodic points of period $t$ ( $M$ is the number of such points). In contrast, the classical search needs $O\left(t N^{2} / M\right)$ operations. As above $M$ can be evaluated through quantum counting in $O(t N)$ operations.

A much studied class of classical twist maps corresponds to the form $\bar{n}=n-K V^{\prime}(\theta)(\bmod 2 \pi L) ; \bar{\theta}=$ $\theta+\bar{n}(\bmod 2 \pi)$ where $(n, \theta)$ is the pair of conjugated momentum (action) and angle variables, and the bars denote the resulting variables after one iteration of the map. The discretized map on a $N \times N$ lattice is simply $\bar{Y}=$ $Y+\left[N K V^{\prime}(2 \pi X / N) /(2 \pi)\right](\bmod N) ; \bar{X}=X+\bar{Y}(\bmod N)$ 
where [...] is the integer part and $X, Y$ are integers. The case $V(\theta)=\cos \theta$ corresponds to the Chirikov standard map which has been a cornerstone model in the study of chaos [17]. The discretized map on a $2^{n_{q}} \times 2^{n_{q}}$ lattice can be implemented in $O\left(n_{q}^{3}\right)$ gates on a quantum computer [8] and therefore a quadratic gain can be achieved for return times and periodic orbits (although other quantities may be obtained with exponential gain [11]). A simpler example is the sawtooth map which corresponds to $V(\theta)=-\theta^{2} / 2$. The discretized mapping is $\bar{Y}=Y+[N K(2 \pi X / N-\pi) /(2 \pi)](\bmod N) ; \bar{X}=$ $X+\bar{Y}(\bmod N)$. This model has been intensively studied in the field of classical chaos [27]. Depending on the values of $K$, the system can be stable or chaotic, and can display complex structures in phase space with chaotic and integrable parts. Interesting phenomena such as anomalous diffusion, self-similar structures, etc... are present. For integer $K$, exponentially large iterates can be computed efficiently and the gain is exponential. For non integer $K$, the iterates cannot be computed efficiently but the map itself can be implemented in $O\left(n_{q}\right)$ gates on a $2^{n_{q}} \times 2^{n_{q}}$ lattice, and a quadratic gain can be achieved for return times and periodic orbits. For example, the case $K= \pm 1 / 2$ for return times requires only three registers (two for $X$ and $Y$, one as workspace) and the algorithm can be realized with as little as 8 qubits and 40 gates per Grover iteration (domain $4 \times 4$ in a $8 \times 8$ lattice).

A particular case arises for systems with symmetries. For example, in the standard and sawtooth maps, the classical evolution operator $L$ is the product of two involutions $L=I_{1} I_{2}$ with $I_{1}^{2}=I_{2}^{2}=1$. Such involutions have lines of fixed points, and it is easy to show that trajectories crossing twice one of these lines are periodic. They correspond to periodic orbits which are invariant through one of the symmetries. These orbits can be found by iterating only points from one of these lines, i.e. $N$ points instead of $N^{2}$. The latter algorithm finds these orbits in $\sqrt{N}$ operations, keeping the quadratic speed-up.

The periodic orbits found by the various algorithms above are exact periodic orbits of the discretized systems, which is a Hamiltonian system in its own right. As concerns the original continuous system, for hyperbolic systems the shadowing theorem [28] ensures that an exact trajectory will remain close to the dynamics of each discretized point for arbitrary times. This exact trajectory will return close to its starting point after the time computed by the algorithm, meaning that it is a Poincaré recurrence time of the system. Moreover, such approximately periodic trajectory can be used as a starting point for a Newton method converging to a true periodic orbit.

In conclusion, it has been shown that on a quantum computer one can obtain Poincaré recurrence times and periodic orbits of certain classical dynamical systems exponentially faster that on a classical computer. For a larger class of systems, a quadratic gain can be achieved.

I thank D. Shepelyansky for many very useful discus- sions, and S. Bettelli and M. Terraneo for critical reading of the manuscript. This work was supported in part by the EC RTN contract HPRN-CT-2000-0156 and by the EC project EDIQIP of the IST-FET programme.

[1] R. P. Feynman, Found. Phys. 16, 507 (1986)

[2] A. Eckert and R. Josza, Rev. Mod. Phys. 68, 733 (1996).

[3] A. Steane, Rep. Progr. Phys. 61, 117 (1998).

[4] M. A. Nielsen and I. L. Chuang, Quantum computation and quantum information, Cambridge Univ. Press, 2000.

[5] P. W. Shor, in Proc. 35th Annu. Symp. Foundations of Computer Science (ed. Goldwasser, S. ), 124 (IEEE Computer Society, Los Alamitos, CA, 1994).

[6] L. K. Grover, Phys. Rev. Lett. 79, 325 (1997).

[7] S. Lloyd, Science 273, 1073 (1996).

[8] B. Georgeot and D. L. Shepelyansky, Phys. Rev. Lett. 86, 2890 (2001).

[9] G. Benenti, G. Casati, S. Montangero and D. L. Shepelyansky, Phys. Rev. Lett. 87, 227901 (2001).

[10] D. A. Lidar and O. Biham, Phys. Rev. E 56, 3661 (1997).

[11] B. Georgeot and D. L. Shepelyansky, Phys. Rev. Lett. 86, 5393 (2001); Phys. Rev. Lett. 88, 219802 (2002).

[12] H. Poincaré, Les méthodes nouvelles de la mécanique céleste, Gauthier-Villars, Paris (1892).

[13] C. Karney, Physica 8D, 360 (1983); B. V. Chirikov and D. L. Shepelyansky, ibid. 13D, 395 (1984); J. Meiss and E. Ott, Phys. Rev. Lett. 55, 2741 (1985); G. Zaslavsky and M. Tippett, ibid. 67, 3251 (1991); B. V. Chirikov and D. L. Shepelyansky, ibid. 82, 528 (1999).

[14] D. Auerbach, P. Cvitanovic, J.-P. Eckmann, G. H. Gunaratne and I. Procaccia, Phys. Rev. Lett. 58, 2387 (1987); P. Cvitanovic, Phys. Rev. Lett. 61, 2729 (1988).

[15] M. C. Gutzwiller, Chaos in classical and quantum mechanics, Springer, N. Y. (1990).

[16] V. I. Arnold and A. Avez, Ergodic Problems of Classical Mechanics, Benjamin, N. Y. (1968).

[17] A. Lichtenberg and M. Lieberman, Regular and Chaotic Dynamics, Springer, N.Y. (1992).

[18] J. H. Hannay and M. V. Berry, Physica 1D, 267 (1980).

[19] F. Vivaldi, Proc. Roy. Soc. London A 413, 97 (1987); I. C. Percival and F. Vivaldi, Physica 25D, 105 (1987).

[20] J. P. Keating, Nonlinearity 4, 277 (1991).

[21] P. Kurlberg and Z. Rudnick, Commun. Math. phys. 222, 1, 201 (2001).

[22] D. Simon, in Proc. 35th Annu. Symp. Foundations of Computer Science (ed. Goldwasser, S. ), 116 (IEEE Computer Society, Los Alamitos, CA, 1994).

[23] V. Vedral, A. Barenco and A. Ekert, Phys. Rev. A 54, 147 (1996); D. Beckman, A. N. Chari, S. Devabhaktuni and J. Preskill, Phys. Rev. A 54, 1034 (1996); C. Miquel, J. P. Paz and R. Perazzo, Phys. Rev. A 54, 2605 (1996).

[24] G. H. Hardy and E. M. Wright, An introduction to the theory of numbers, Oxford University Press, Oxford $\left(5^{\text {th }}\right.$ Ed., 1979).

[25] F. Rannou, Astron. Astrophys. 31, 289 (1974); D. J. D. Earn and S. Tremaine, Physica D 56, 1 (1992). 
[26] M. Boyer, G. Brassard, P. Høyer and A. Tapp, Fortschr. Phys. 46, 493 (1998).

[27] I. Dana, N. W. Murray and I. C. Percival, Phys. Rev. Lett. 62, 233 (1989); Q. Chen, I. Dana, J. D. Meiss, N. W. Murray and I. C. Percival, Physica 46 D, 217 (1990).

[28] D. V. Anosov, Proc. Steklov. Inst. Math. 90,1 (1967); R. Bowen J. Diff. Eq. 18, 333 (1975); T. Sauer, C. Grebogi and J. A. Yorke, Phys. Rev. Lett. 79, 59 (1997). 